\title{
Protective effect of Soya Bean oil against Bisphenol A induced interstitial lung fibrosis in mice.
}

1. MBBS, M.Phil (Anatomy) Assistant Professor Anatomy Rehman Medical College Peshawar.

2. MBBS, M.Phil (Anatomy) Assistant Professor Anatomy Army Medical College.

3. MBBS, M.Phil (Anatomy) Assistant Professor Anatomy Army Medical College.

4. MBBS

Assistant Professor Pharmacology Rehman Medical College Peshawar.

Correspondence Address:

Dr. Sadia Shaukat

Department of Anatomy

Rehman Medical College Peshawar.

sadia.shaukat@rmi.edu.pk

Article received on:

30/01/2021

Accepted for publication:

$30 / 03 / 2021$
Sadia Shaukat ${ }^{1}$, Faiza Umbreen ${ }^{2}$, Humaira $\mathrm{Ali}^{3}$, Aamna Khokhar ${ }^{4}$

ABSTRACT... Objective: The objective of this study was to evaluate cytoprotective effects of soyabean oil supplementation against interstitial fibrosis induced in lungs of adult mice by Bisphenol A. Study Design: Experimental Study. Setting: Department of Anatomy, Army Medical College, Rawalpindi. Period: Nov 2015 to Nov 2016. Material \& Methods: Forty healthy adult BALB/c mice of $9-11$ weeks of age and weighing from $30-37 \mathrm{gms}$ were included in this study and were housed in controlled environment of animal house of $\mathrm{NIH}$, Islamabad. Group A animals (10) served as controls. Group B animals (10) were given BPA orally at a dose of $50 \mathrm{mg} /$ $\mathrm{kg}$ body weight/day and Group C animals (10) were given soya bean oil at a dose of $500 \mathrm{mg} / \mathrm{day}$ and Group D animals (10) were given BPA and Soya bean oil at doses of $50 \mathrm{mg} / \mathrm{kg}$ body weight/ day and $500 \mathrm{mg} /$ day respectively. All doses were administered orally once daily for a period of eight weeks. Animals were dissected $24 \mathrm{hrs}$ after receiving the last dose. Lung tissue specimen processing and Masson trichrome staining was done for the histological study. Lung interstitial fibrosis was morphometrically and statistically analysed using SPSSv21. Results: Microscopic examination showed Grade 2 interstitial fibrosis in $80 \%$ specimens in Group B, whereas only $60 \%$ specimens of Group D had Grade 1 interstitial fibrosis. Conclusion: It was concluded from results that BPA produces interstitial fibrosis and con-concomitant administration of soya bean oil diet protects against development of lung interstitial fibrosis.

Key words: Bisphenol A, Interstitial Fibrosis, Soya Bean Oil.

Article Citation: Shaukat S, Umbreen F, Ali H, Khokhar A. Protective effect of soya bean oil against Bisphenol $A$ induced interstitial lung fibrosis in mice. Professional Med J 2021; 28(10):1457-1462.

https://doi.org/10.29309/TPMJ/2021.28.10.6391

\section{INTRODUCTION}

Interstitial lung fibrosis, scarring of the lung affects millions of people worldwide. In Europe and North America alone, reported incidence of IPF is between 2.8 and 19 cases per 100000 people per year. ${ }^{1}$ It includes idiopathic disease, pneumoconiosis/particle-induced disease or inflammatory processes. Lung fibrosis exhibits a diverse aetiology and prognosis including inhaled agents, environmental or occupational exposure to particles, organic dusts, and family history.

Bisphenol A (BPA) or 2'-bis (4-hydroxyphenyl) propane bearing the chemical formula $\left(\mathrm{CH}_{3}\right)_{2} \mathrm{C}$ $\left(\mathrm{C}_{6} \mathrm{H}_{4} \mathrm{OH}\right)_{2}$ was first discovered by Edward Charles Dodds. BPA exhibits estrogenic properties ${ }^{2}$, acts as inflammation promoting factor acting through estrogen receptor $\beta$ (ER $\beta){ }^{3}$ Significant studies have proved effects of low or high doses of BPA in experimental animals, causing considerable structural and functional impairment in lungs as well as prostate, testis, mammary gland, uterus, cardiovascular system and malformations of genitalia, along with modifications in brain structure, chemistry and behaviours., ${ }^{4,5}$ BPA disrupts STAT3, MAPK, and PI3K/AKT oncogenic signaling pathways leading to carcinogenic effects. ${ }^{6}$ These revelations raised safety concerns about use of BPA in the manufacturing of beverage and food contact containers resulting in prohibition of use of BPA in plastic feeding bottles and infant powdered formula packaging materials in $2013^{5}$ by the Food and Drug Administration USA (FDA). TDI (tolerable daily intake) of BPA has been reduced from $50 \mu \mathrm{g} /$ $\mathrm{kg} /$ day to $4 \mu \mathrm{g} / \mathrm{kg} /$ day, with the highest level of dietary exposure being documented to be three to five times lower than the new TDI. Michałowicz ${ }^{7}$ 
investigated human exposure to BPA by consumption of Bisphenol A contaminated food or water in addition to exposures through digital receipts, electronic equipment, automobiles, safety equipment and paints. Occupational exposures are predominantly via inhalation and dermal routes. ${ }^{8}$ Chronic BPA exposure especially to its metabolite 4-Methyl-2,4-bis(4hydroxyphenyl) pent-1-ene alters lung function and growth of surfactant producing pneumocyte cells, leading to pulmonary inflammatory diseases and lung interstitial fibrosis., 90 BPA modifies development, maturation and functioning of lungs during prenatal and postnatal life, leading to asthma and allergic lung inflammation especially during childhood. ${ }^{11,12}$

Soyabeans contain abundant bioactive plant compounds including phytic acid, isoflavones and saponins. Isoflavones are antioxidant polyphenols (also known as phytoestrogens) ${ }^{13}$, having weak estrogenic and antioxidant activity. ${ }^{14}$ Research has substantiated that Phytic acid in soyabean oil also has antioxidant and chelating properties. ${ }^{15}$ Saponins are cholesterol lowering agents. ${ }^{16}$ In addition, molybdenum, vitamin $\mathrm{K} 1$, folate, copper, manganese, phosphorus, and thiamine are also present in significant amounts.

Majority of studies on BPA focus on either endocrine disruptions or the embryonic reproductive system. However, tissue distribution of BPA in rats is predominantly to the lungs. Hence, objective of this study was to study the evaluate the effect of BPA on the lung interstitium of adult mice and to assess the potential protective role of soyabean oil supplementation in the BPA induced lung fibrosis.

\section{MATERIAL \& METHODS}

Randomized control study of one-year duration was carried out in Department of Anatomy, Army Medical College (AMC) Rawalpindi in collaboration with Pathology Department and National Institute of Health (NIH) from November 2015 to November 2016. The study was conducted after ethical approval of authorities of Army Medical College, (ERC/SA-16/Dr. Sadia Shaukat). Rawalpindi. The experimental chemical
Bisphenol A was purchased from Sigma Eldritch.

Forty adult BALB/c mice, 9-11weeks old and weighing $30-37 \mathrm{gm}$ were included in this experiment. Mice were kept in polystyrene cages in a well-ventilated room of $\mathrm{NIH}$ with room temperature ranging from $20-26^{\circ} \mathrm{C}$ and were permitted 12hours of dark-light sleep cycle. ${ }^{11}$ Mice were fed with Soya bean free lab diet (to remove phytoestrogen content of pallets) and minimize background BPA exposure. ${ }^{4,17}$ Water was supplied ad libitum in polystyrene bottles. All doses were administered via oral gavage once daily for a total period of eight weeks.

Mice in Group A served as untreated controls. Experimental Group B was administered with $50 \mathrm{mg} / \mathrm{kg} /$ day of BPA. Group C was administered with $500 \mathrm{mg} /$ day of soyabean oil and mice in experimental Group D were administered 50mg/ $\mathrm{kg} /$ day of BPA and $500 \mathrm{mg} /$ day of soyabean oil simultaneously. At the end of 8-week experiment, animals were euthanized; sacrificed, dissected and fresh lung specimens were removed. Lung tissue processing was done in 10 per cent formalin and 5-micron thick sections were cut using rotary microtome. Masson's trichrome staining was used to demonstrate deposition of supporting collagen fibre in alveolar epithelium, capillary endothelium as well as in perivascular and peribronchial areas. Slides were examined under 40X for the presence of fibrosis on a semi quantitative score described ${ }^{18}$ as follows:

Grade 0: normal lung, Grade 1: isolated septa with gentle fibrotic changes with septa $<3$ times thicker than normal and minimal fibrous thickening of bronchiolar walls, Grade 2: Clear fibrotic changes of alveolar septa being $>3$ times thick with knot-like formation not connected to each other, Grade 3: contiguous fibrotic walls of alveolar septa, Grade 4: single fibrotic masses.

SPSS version 21 was used for data analysis. Qualitative variables were expressed as frequency and percentage and compared by Chisquare test. $\mathrm{P}$-value of $<0.05$ was considered significant. 


\section{RESULTS}

In all $10(100 \%)$ specimens of control Group A, normal lung tissue was seen, without fibrosis. In experimental Group B, 2(20\%) of specimens showed Grade 2 fibrotic changes and $8(80 \%)$ had Grade 3 fibrotic changes (Table-I, Figure-2b). On intergroup comparison, Group B was statistically significant with Group D ( $p$-value $=0.001)$ and highly significant with control Group $A$ and experimental Group C ( $p$-value $<0.0001$ ) (Table-I, Figure-1). In experimental Group C, fibrosis was recorded at Grade 0 in $8(80 \%)$ of specimens whereas 2(20\%) specimens showed Grade-1 fibrosis (Table-I, Figure-2a). On comparison of experimental Group C with experimental
Group B ( $p$-value $<0.0001)$, results were highly significant ( $p$-value $<0.0001)$ and the difference was statistically significant when compared with experimental Group D ( $p$-value $=0.001)$, but insignificant when compared with Group A $(p$-value $=0.474) \quad($ Table-I, Figure-1). Whereas in Experimental Group D, 6(60\%) of the specimens showed Grade 1 infiltrates, 3(30\%) specimens showed Grade 2 (Table-l, Figure-2c) and rest of the $1(10 \%)$ had Grade 3 fibrosis. On intergroup comparison, it was found to be statistically significant when compared with control Group $A$, experimental Group D ( $p$-value $<0.0001)$ and experimental Groups C ( $p$-value $=0.001)$ (Table-I, Figure-1).

\begin{tabular}{|l|c|c|c|c|c|c|}
\hline Parameters & Findings & Group A & Group B & Group C & Group D & P-Value \\
\hline & Grade 0 & $10(100 \%)$ & $0(0.0 \%)$ & $8(80 \%)$ & $0(0.0 \%)$ & $<0.0001^{*}$ \\
\cline { 2 - 7 } & Grade 1 & $0(0.0 \%)$ & $0(0.0 \%)$ & $2(20 \%)$ & $6(60 \%)$ & 0.474 \\
\cline { 2 - 7 } Interstitial fibrosis & Grade 2 & $0(0.0 \%)$ & $2(20 \%)$ & $0(0.0 \%)$ & $3(30 \%)$ & $<0.0001^{*}$ \\
\cline { 2 - 7 } & Grade 3 & $0(0.0 \%)$ & $8(80 \%)$ & $0(0.0 \%)$ & $1(10.0 \%)$ & $<0.0001^{*}$ \\
\cline { 2 - 7 } & Grade 4 & $0(0.0 \%)$ & $0(0.0 \%)$ & $0(0.0 \%)$ & $0(0.0 \%)$ & 0.001 \\
\hline
\end{tabular}

Table-I. Frequency and percentages of interstitial fibrosis of control group A and experimental groups B, C and D $(n=40)$.

$\mathrm{p}$-value $\leq \mathbf{0 . 0 5}$ is statistically significant $\quad *=$ highly significant



Figure-1. Cluster Bar chart showing comparison of frequency of interstitial fibrosis with scoring among the control group A and experimental groups B, C and D $(n=40)$.

\section{DISCUSSION}

The wide spread use of BPA-containing manufactured goods has caused concern because of harmful effects on various vital organs. Accordingly, this study was designed to study the effects of BPA on mice lungs along with administration of soybean oil that can have protective effect.

Masson's trichrome stained slides were examined to observe fibrotic changes in the interstitial space around bronchi, vessels and in the inter alveolar septa using Ashcroft score. In the present study, Group B was found to be highly significant as compared to Control Group $A$ and experimental Group C with p-value $<0.0001$. Group B was also significant when compared with Group D with $p$-value $=0.001$. Similar effects of Chronic exposure to oral BPA on lungs were documented by Kattaia, in 2014, Egypt as a result of inflammation and oxidative stress by producing increased levels of Interleukin-18 (IL18), malondialdehyde (MDA), and decline in levels of superoxide dismutase (SOD)..$^{19}$

The current work proposed increase in positive immunoreaction for INOS and formation of reactive oxygen species (ROS) leading to oxidative stress. 


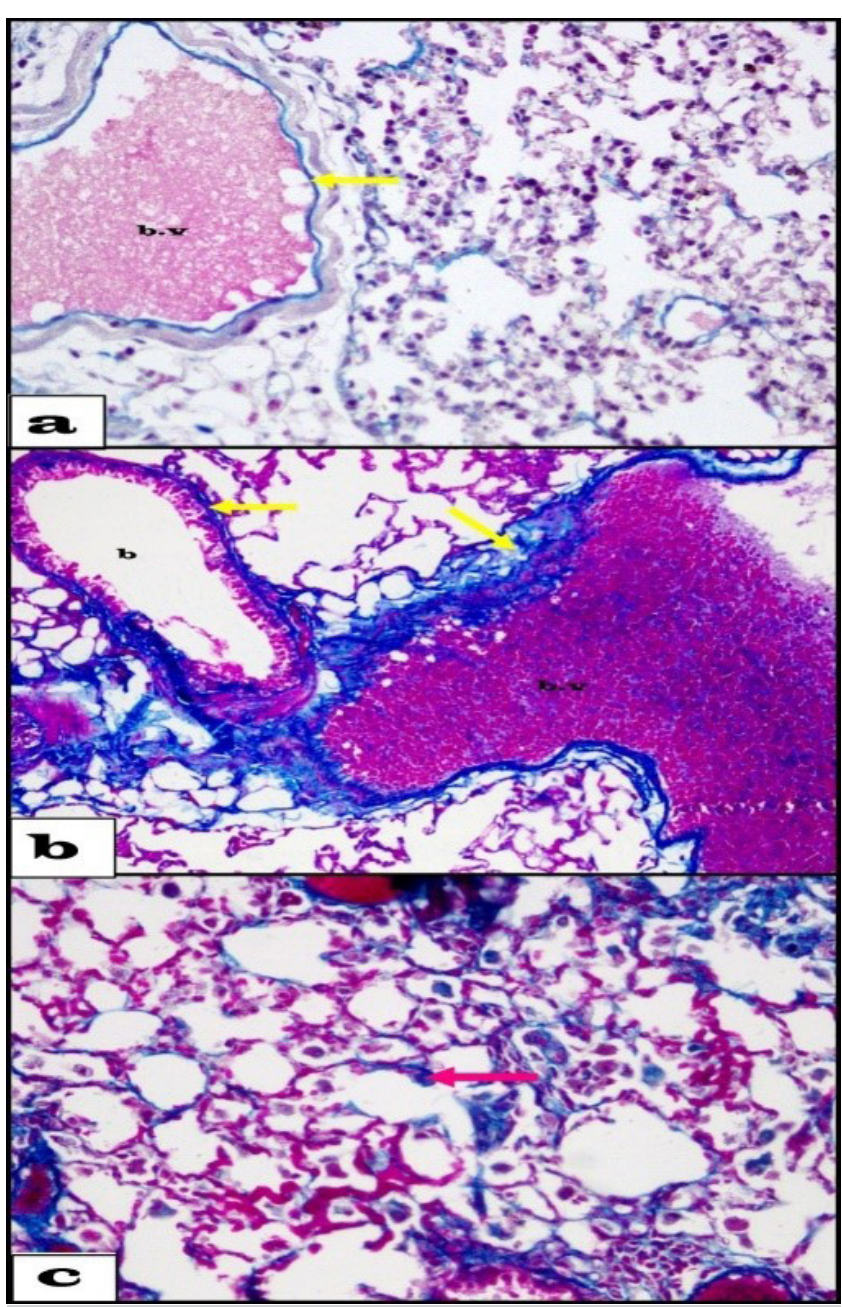

Figure-2. Photomicrograph showing interstitial fibrosis using Ashcroft score. Animal no.7 of experimental Group C showing Grade 1 fibrosis, (b) animal no.1 of experimental Group B showing Grade 3 fibrosis and (c) animal-2 of experimental Group D showing Grade 2 fibrosis. Collagen fibres (yellow arrow), Knot formation (pink arrow): 40X, Masson Trichrome.

Karnam, in 2016, India also suggested oxidative stress leading to various lung conditions including congestion, inflammation, alveolar hemorrhage, and diffuse increase in histocytes in animals treated with BP A. In addition, increased levels of pro-fibrotic IL-13 and/or TGF $\beta 1$ factors are produced, ultimately disrupting the healing process and culminating into a pathogenic fibrotic reaction. ${ }^{20}$ Acute inflammatory processes also result in fibroblastic proliferation and synthesis of extracellular collagen matrix producing fibrosis, which is characterized by thickened alveolar septal walls and diminished air spaces. ${ }^{18}$ BPA intensifies collagen fibre deposition in a dose dependant manner involving above mentioned processes and by activation of a transcription factor KB with resultant fibrosis or apoptosis. In addition, genes associated with fibrosis demonstrate increased expression of follistatinlike1 (FSTL1) factors and concomitant decrease in 5 (ADAMTS5) expression levels ${ }^{9}$ intermediating proteolysis and loss of aggrecan.

It was further demonstrated by Liuand other researchers in 2016 that 4-Methyl-2,4-bis(4hydroxyphenyl)pent-1-ene (MBP) a major active metabolite of BPA induces apoptosis of surfactant producing pneumocyte cells and consequently lung dysfunction by enhancing AMP-activated protein kinase (AMPK) phosphorylation and molecular expression of AMPK-regulated ER and endoplasmic reticulum (ER) stress-signalling pathway in pulmonary epithelial cell line L2 of adult rats. ${ }^{10}$

Experimental Group D specimens showed ameliorative effects with $60 \%$ falling in Grade 1, $30 \%$ in Grade 2 and only $10 \%$ in Grade 3, validating ameliorative effects of Soya bean oil. Studies have shown that in non-small-cell lung cancer (NSCLC), adverse effects of radiation including inflammation, pneumonitis and fibrosis can be greatly reduced by soy is flavones consumption during pre and post-radiation period. ${ }^{21}$ Portal C, in 2018, France proposed that Soya bean with abundant long-chain polyunsaturated fatty acids (LC-PUFAs) reduce hyperplasia and lung inflammation of bronchial epithelial cells, thus restricting oxidative stress and consequently lung damage. ${ }^{22}$

\section{CONCLUSION}

It was observed in present study that oral administration of BPA causes fibrotic changes in lung interstitium of adult mice and that concurrent administration of soyabean oil can counteract the fibrotic changes. Caused by BPA.

\section{ACKNOWLEDGEMENT}

We would like to thank our supervisor and associate Professor of Department of anatomy, AMC, Brig. Shabnum Hamid for her guidance and constant help throughout my work. 
Copyright $\odot 30$ Mar, 2021.

\section{REFERENCES}

1. Olson AL, Gifford AH, Inase N, Pérez ERF, Suda TJERR. The epidemiology of idiopathic pulmonary fibrosis and interstitial lung diseases at risk of a progressivefibrosing phenotype. 2018; 27(150):180077.

2. Dodds EC, Lawson W. Molecular structure in relation to oestrogenic activity. Compounds without a phenanthrene nucleus. Proceedings of the Royal Society of London B: Biological Sciences. 1938; 125(839):222-32.

3. Hijazi A, Guan H, Yang KJAoT. Bisphenol A suppresses glucocorticoid target gene (ENaCy) expression via a novel $E R \beta / N F-k B / G R$ signalling pathway in lung epithelial cells. 2017; 91(4):1727-37.

4. Karnam S, Ghosh R, Mondal MJIJoVP. Pathology of bisphenol $A$ induced sub-acute toxicity in Albino rats. $2016 ; 40(1): 47-50$.

5. Sciacca S, Conti GO, Fiore M, Ferrante M. Focus on Bisphenol A, an Uncertain Environmental Pollutant. Edited by Margarita Pesheva, Martin Dimitrov and Teodora Stefkova Stoycheva. 2012:69.

6. Gao H, Yang B-J, Li N, Feng L-M, Shi X-Y, Zhao W-H, et al. Bisphenol A and hormone-associated cancers: current progress and perspectives. Medicine. 2015; 94(1).

7. Michałowicz J. Bisphenol A-sources, toxicity and biotransformation. Environmental toxicology and pharmacology. 2014; 37(2):738-58.

8. Tsai W-T. Human health risk on environmental exposure to bisphenol-A: a review. Journal of Environmental Science and Health Part C. 2006; 24(2):225-55.

9. Abedelhaffez AS, El-Aziz EAA, Aziz MAA, Ahmed AMJP. Lung injury induced by Bisphenol $A$ : $A$ food contaminant is ameliorated by selenium supplementation. $2017 ; 24(2): 81-9$.

10. Liu S-H, Su C-C, Lee K-I, Chen Y-W. Effects of bisphenol a metabolite 4-methyl-2, 4-bis (4-hydroxyphenyl) pent-1-ene on lung function and type 2 pulmonary alveolar epithelial cell growth. Scientific Reports. 2016; 6:39254.

11. Donohue KM, Miller RL, Perzanowski MS, Just AC, Hoepner LA, Arunajadai S, et al. Prenatal and postnatal bisphenol A exposure and asthma development among inner-city children. Journal of Allergy and Clinical Immunology. 2013; 131(3):736-42. e6.
12. Van Winkle LS, Murphy SR, Boetticher MV, Vande Voort CAJE hp. Fetal exposure of rhesus macaques to bisphenol a alters cellular development of the conducting airway by changing epithelial secretory product expression. 2013; 121(8):912-8.

13. Achouri A, Boye Jl, Belanger D. Soybean is flavones: Efficacy of extraction conditions and effect of food type on extractability. Food Research International. 2005; 38(10):1199-204.

14. Teixeira $C$, Simões $R$, Santos $M$, Calió $M$, Soares Jr $J$, Simões $M$, et al. Soybean concentrated extract counteracts oxidative stress in the uterus of rats. Climacteric. 2014; 17(4):402-9.

15. Kumar MS, Reddy BS, Babu SK, Bhilegaonkar P, Shirwaikar A, Unnikrishnan M. Anti-inflammatory and antiulcer activities of phytic acid in rats. Indian journal of experimental biology. 2004; 42(2):179-85.

16. Lee SO, Simons AL, Murphy PA, Hendrich S. Soyasaponins lowered plasma cholesterol and increased fecal bile acids in female golden Syrian hamsters. Exp Biol Med. 2005; 230(7):472-8.

17. Kattaia AA, Baset SAA. Effect of bisphenol $A$ on the lung of adult male albino rats and the possible protective role of geraniol: a histological and immunohistochemical study. Egyptian Journal of Histology. 2014; 37(1):24-35.

18. Hubner R, Gitter W, El Mokhtari NE, Mathiak M, Both $\mathrm{M}$, Bolte $\mathrm{H}$, et al. Standardized quantification of pulmonary fibrosis in histological samples. Biotechniques. 2008; 44(4):507.

19. Kattaia AA, Baset SAAJEJoH. Effect of bisphenol A on the lung of adult male albino rats and the possible protective role of geraniol: a histological and immunohistochemical study. 2014; 37(1):24-35.

20. Wilson M, Wynn T. Pulmonary fibrosis: pathogenesis, etiology and regulation. Mucosal immunology. 2009; 2(2):103.

21. Hillman GG, Singh-Gupta V, Lonardo F, Hoogstra DJ, Abernathy LM, Yunker CK, et al. Radioprotection of lung tissue by soy isoflavones. Journal of Thoracic Oncology. 2013; 8(11):1356-64.

22. Portal C, Gouyer V, Leonard R, Husson M-O, Gottrand F, Desseyn J-L. Long-term dietary (n-3) polyunsaturated fatty acids show benefits to the lungs of Cftr F508del mice. PloS one. 2018; 13(6):e0197808. 


\section{AUTHORSHIP AND CONTRIBUTION DECLARATION}

\begin{tabular}{|c|l|l|}
\hline Sr. \# & Author(s) Full Name & \multicolumn{1}{|c|}{ Contribution to the paper } \\
\hline 1 & Sadia Shaukat & $\begin{array}{l}\text { Concept, design of work, Acquisition, } \\
\text { analysis and interpretation of data, } \\
\text { critical revision of work. } \\
\text { Contribution to critical revision of } \\
\text { work, final approval of revision of } \\
\text { work. } \\
\text { Contributed in collections, analysis } \\
\text { and interpretation of data. } \\
\text { Dosage calculation and } \\
\text { pharmacological aspect of work. }\end{array}$ \\
\hline 3 & Faiza Umbreen & Aamna Khokhar
\end{tabular}

